\title{
A Design of an Experimental System for Trapping Pressure of Agricultural Gear Pump
}

\author{
Xiangdong Zhu*, Kejiang Zang, Guoling Niu, and Xiaohai Li \\ College of Mechanical Engineering, Jiamusi University, Jiamusi, \\ Heilongjiang Province, P.R. China 154007, \\ Tel.: 0454-8763218 \\ zhuxiangdong_jms@163.com
}

\begin{abstract}
The trapping phenomenon stems from the principle of gear pumps, and it affects directly the performance and life of gear pump. Reducing pressure in trapping area is an important aspect to improve working performance of gear pump, and how to test the pressure of trapping area is one of the most important problems. According to such requirements, an experimental system has been designed, and then relevant experiments are conducted on the designed experimental system. The experimental results indicate that the system can fulfill the requirements commendably.
\end{abstract}

Keywords: gear pump, trapping, oil pressure, testing system.

\section{Introduction}

In order to make gear teeth of gear pumps mesh well, and make inlet chamber and outlet chamber seal tightly for exporting working medium evenly and continuously, the overlap coefficient must be more than one $(\varepsilon>1)$. In other words, before one pair of teeth is about to stop meshing, another pair of teeth has meshed. More than one pair of teeth mesh simultaneously. Generally, there is a sealed space formed by two pairs of gear teeth, and the oil can be trapped in the sealed space. The sealed space is called trapping chamber. But the sealed space will turn smaller and smaller along with the pump work continuously. When the two meshing nodes locate the symmetrical position of two pairs of gears' notes, the sealed space will be reduced to the smallest cubage. Since the oil compressibility property is small, the oil pressure turns high rapidly for the decrease of the cubage of the sealed space. When the oil pressure exceeds far the outlet chamber pressure, the oil is extruded from the both sides of the adjoining plane parts. So the shafts and bearings of the gear pump endure heavy shock load, and make power loss increase, medium heat, vibration and noise produce. Thus the work stability and the life of the pump will be decreased. As gears continue to mesh, the trapping chamber cubage turns bigger and bigger, so the vacuum will be formed. Bubbles, cavitations, vibration, noise and other hazards appear subsequently. This impact load change periodically along with alteration of the trapping space

\footnotetext{
${ }^{*}$ Corresponding author.
} 
volume in turn. And moreover the original stack load will be superimposed. Although the trapping phenomenon is happened shortly, its frequency is very high. So the alternating load has a periodic property.

The above is a general description of the trapping phenomenon. In fact, under the environment of the micro-clearance gear meshing or anti-backlash gear meshing, when a pair of teeth meshes, the trapping phenomenon will also occur. There are two trapping chambers are formed under a pair teeth meshing, and three trapping chambers are formed under two pairs teeth meshing. The trapping phenomenon may affect the life of pump and the working performance badly.

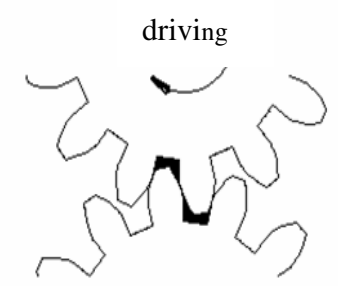

a. A pair of teeth meshin

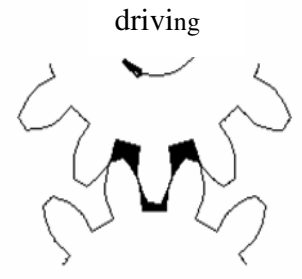

b. two pair of teeth meshing

Fig. 1. Trapping area

Reducing the trapping pressure of gear pump is one of the most important aspects to improve the pump's work performance. Home and abroad scholars have done large amount of job on it. Among them, unloading grooves is the most effective method and it is applied broadly. So many theoretical analyses have done on the trapping pressure but the problem that how to test the pressure is rarely reported. A test method that has mentioned in the document (Zhao Liang, et al., 2004) is difficult to carry out. In recent years, the author studies on how to reduce the trapping pressure of gear pump and designs an experiment test system to test the trapping pressure change. Following content introduce this experiment system only for reference.

\section{The Principle of Unload-Pressure-Relief Groove}

(1) work surface and non-work surface

Gear tooth has two figure surfaces. One is meshing surface for the sake of transferring power, the other is towards it for the sake of holding balanced transferring. In order to narrate easily, we called the former "work surface" and the latter "non-work surface".

(2) unload-pressure-relief groove

The unload-pressure-relief groove is a groove on the not work surface, show as Fig.2. As a result of radial groove, it can not destroy meshing and intensity of the work surface, and stability is invariable.

(3) Principle

The principle of the unload-pressure-relief groove is show as Fig.3, which not setting pressure unloading grooves, supposing meshing non-profile clearance. From 


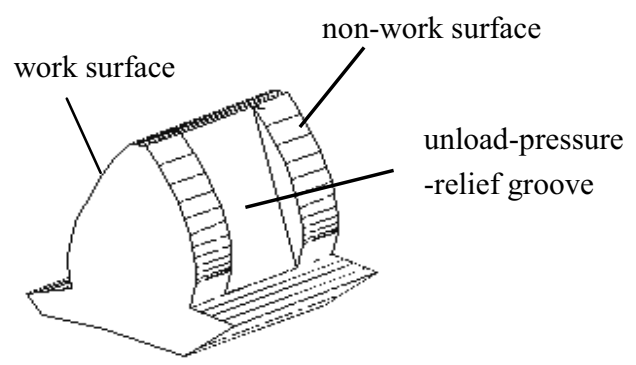

Fig. 2. Gear tooth with the unload-pressure-relief groove
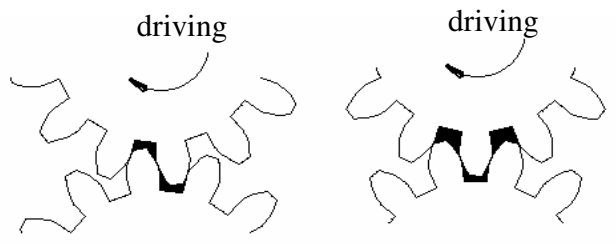

(a)gear pump without the unload-pressurerelief groove
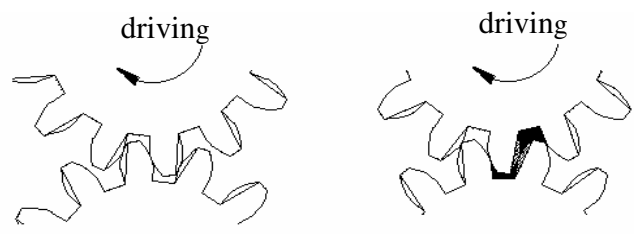

(b) gear pump with the unload-pressure-relief groove

$\mathbf{\square}$ : the trapping volume without the unload-pressure-relief groove

䍘: the additive trapping volume with the unload-pressure-relief groove

Fig. 3. Relation of gear pump without the unload-pressure-relief groove and with the unloadpressure-relief groove

the Fig.3, we can see that a pair of gear teeth is meshing, then two trapping chambers are formed when the unload-pressure-relief grooves are not set; while there isn't trapping chamber when unload-pressure-relief grooves are set. Two pairs of gear teeth meshing, there are three trapping chambers, when the unload-pressure-relief grooves are not set; while there are two trapping chambers when the unload-pressure-relief grooves are set, moreover they are connected. In general, the numbers of trapping chambers are reduced after the unload-pressure-relief grooves are set. 


\section{Experiment Test System}

The experiment test system is shown in Fig.4. A volume flow control circuit is built with a volume adjustable hydraulic pump 3 and a single-way constant displacement motor 6 . The tested pump 7 is driven by the single-way constant displacement motor. The tested pump's speed can be adjusted by changing volume adjustable hydraulic pump during the test. Bypass valve 5 is used as a safety valve and throttle valve 9 is used for adjusting the tested gear pump's work pressure. Motor drives the tested pump through a coupler. The apparatus 8 is speed transducer, which measure the tested pump's operating rotational speed, two pressure transducers are installed at the two ends of tested pump for testing the trapping pressure change. Datum getting from the pressure transducers and speed transducers is collected by the datum acquiring meter 10 and written into the recording equipment. Finally, through soft ware in the computer disposes the datum to obtain the data and the graph that needed (H Yanada, et al., 2006).

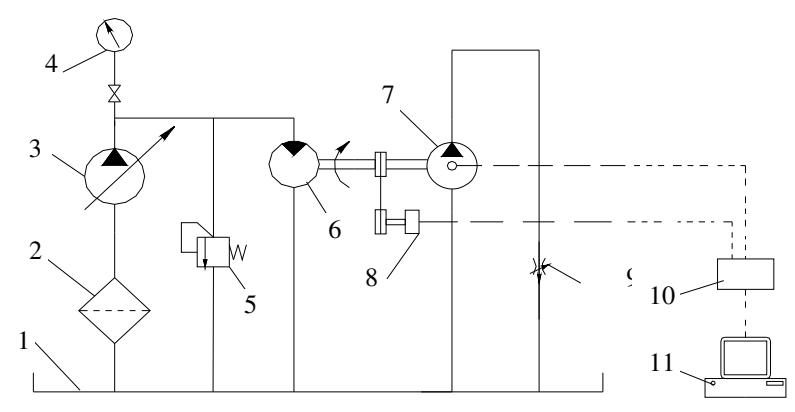

1. tank 2. filter 3. variable displacement pump 4. pressure meter 5. relief valve 6. fixed displacement motor 7. tested gear pump 8. speed transducer 9.check valve 10. datum collecting instrument 11. recording equipment (computer)

Fig. 4. Experiment test system sketch

\section{The Design of the Tested Gear Pump}

The aim of this experiment is to test the pressure alternation of the pump's trapping pressure for studying the effects of different measures, which are taken to decrease the pressure of trapping chamber, so the structure of tested pump is three pieces types structure, which is shown in Fig.5. The tested pump mainly consists of the back cover 1, pump body 4 , front cover 6 , the driving gear 7 and driven gear 5 . Inlet port and outlet port are installed in the back cover. The structure of the tested pump is similar with CBB gear pump. The driven gear's design is a key part. In order to get the pressure signals from the ends of the driven gear, so the driven gear and driven shaft are designed one body. At the end of shaft the trapping area pressure signals are got from axial holes and radial holes, in the driven gear and driven shaft and are tested by the pressure transducer 3. The sketch of gears' structure and assembly is shown in Fig.5. 

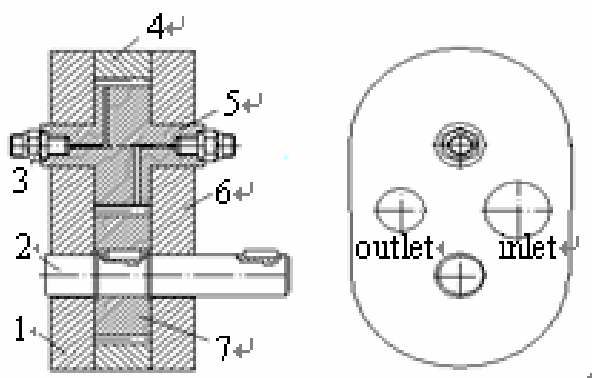

1. back cover 2. driver shaft 3 . pressure transducer 4. pump body 5 . driven gear 6 . front cover

7. driver gear

Fig. 5. Structure of the tested gear pump

\section{Test Experiment Example}

According to the above test principle, the test equipment had been developed to test the trapping pressure in a tested gear pump show as Fig.6. The experiment of measuring trapping pressure change is carried out. The conditions of the tested gear pump are shown as following.

Table 1. Structural parameters of gear pump

parameter modulus Tooth number press angle tooth width tooth top parameter tooth top clearance parameter

$\mathrm{mm} \quad \operatorname{deg} \quad \mathrm{mm}$

$\begin{array}{lllllll}\text { Value } & 5 & 13 & 20 & 35 & 1 & 0.25\end{array}$

The pressure transducer model is BPR-2, which is connected with datum collecting instrument whose model is WS-U20116 [B] by an electric resistance strain instrument. The pressure value of trapping area is collected continuously, and the datum collected can be put into computer directly and disposed by the collecting instrument datum handling system. Fig. 4 shows trail curve of pressure ( $n=200 \mathrm{r} / \mathrm{min})$. Since the datum are measured continuously, so the pressure curves reflect not only the trapping pressure alteration but also the alteration of the inlet chamber pressure, the transition region pressure, the outlet chamber pressure. Here the peak value area of the curve expresses trapping area's pressure. 


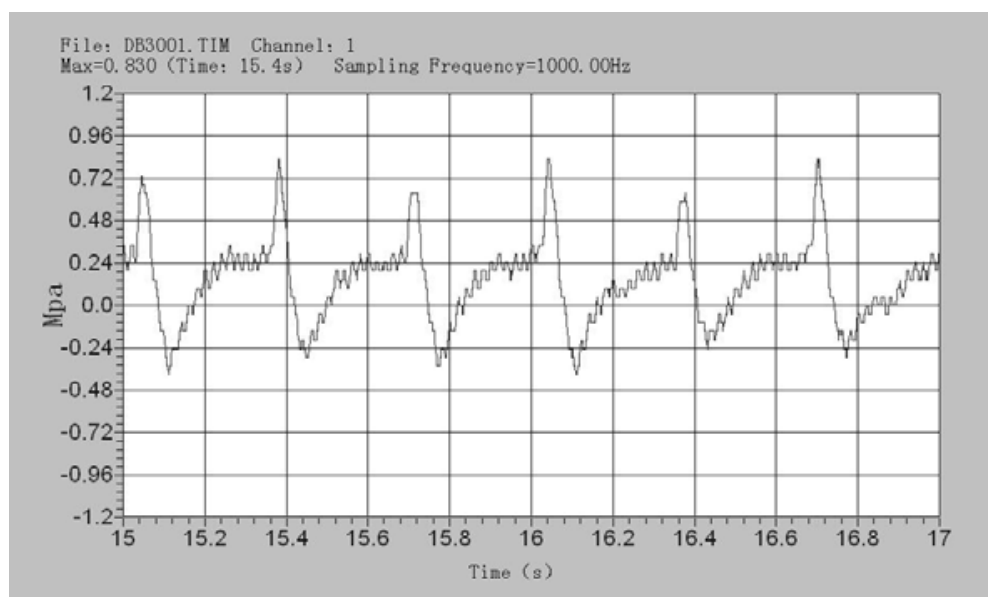

Fig. 6. Trail curve of pressure/time $(n=200 \mathrm{r} / \mathrm{min})$

\section{Conclusions}

In this experimental test system, the pressure signals were obtained from the driven shaft's end. The test points following with the driven gear traveled inlet chamber, the transition region, and outlet chamber. The pressure curves which we obtained from the points not only included the trapping pressure alteration but also obtained the inlet chamber pressure, the transition region pressure, the outlet chamber pressure. So gear pump's working performance can be studied from the pressure curves.

\section{Acknowledgements}

The authors would like to thank the project (10541223) supported by Scientific Research Fund of Heilongjiang Province Education Department.

\section{References}

Liang, Z., Xiyan, R., Dong-ping, W.: Analysis of gear pump for trapping in a very small clearence between gears. Chinese Journal of Mechanical Engineering 35(6), 77-80 (2005) (in Chinese)

Yanada, H., Ichikawa, T., Itsuji, Y.: Study on the phenomenon oil-trap stems of gear pump. Chinese Hydraulics \& Pneumatics (3), 47-52 (2006) (in Chinese) 